\title{
Genome-wide analysis and prediction of functional long noncoding RNAs in osteoblast differentiation under simulated microgravity
}

\author{
ZEBING HU $^{1 *}$, HAN WANG $^{1,2^{*}}$, YIXUAN WANG ${ }^{1}$, HUA ZHOU $^{1}$, FEI SHI $^{1}$, \\ JIANGDONG ZHAO $^{1}$, SHU ZHANG ${ }^{1}$ and XINSHENG CAO ${ }^{1}$ \\ ${ }^{1}$ The Key Laboratory of Aerospace Medicine, Ministry of Education, The Fourth Military Medical University, \\ Xi'an, Shaanxi 710032; ${ }^{2}$ Department of Orthopaedics, Affiliated Hospital of \\ Air Force Aviation Medicine Research Institute, Beijing 100089, P.R. China
}

Received March 1, 2017; Accepted August 17, 2017

DOI: $10.3892 / \mathrm{mmr} .2017 .7671$

\begin{abstract}
Long noncoding RNAs (lncRNAs) have been regarded as important regulators in numerous biological processes during cell development. However, the holistic lncRNA expression pattern and potential functions during osteoblast differentiation under simulated microgravity remain unknown. In the present study, a high throughput microarray assay was performed to detect lncRNA and mRNA expression profiles during MC3TC-E1 pre-osteoblast cell osteo-differentiation under simulated microgravity. The expression of 857 lncRNAs and 2,264 mRNAs was significantly altered when MC3T3-E1 cells were exposed to simulated microgravity. A relatively consistent distribution pattern on the chromosome and a co-expression network were observed between the differentially-expressed lncRNAs and mRNAs. Genomic context analysis further identified 132 differentially-expressed lncRNAs and nearby coding gene pairs. Subsequently, 3 lncRNAs were screened out for their possible function in osteoblast differentiation, based on their co-expression association and potential cis-acting regulatory pattern with the deregulated mRNAs. The present study aimed to provide a comprehensive understanding of and a foundation for future studies into lncRNA function in mechanical signal-mediated osteoblast differentiation.
\end{abstract}

Correspondence to: Professor Shu Zhang or Professor Xinsheng Cao, The Key Laboratory of Aerospace Medicine, Ministry of Education, The Fourth Military Medical University, 169 Changle West Road, Xi'an, Shaanxi 710032, P.R. China

E-mail: shuzhang89@hotmail.com

E-mail: caoxinsh@fmmu.edu.cn

*Contributed equally

Key words: osteoblast differentiation, long noncoding RNA, simulated microgravity, bioinformatic analysis

\section{Introduction}

Studies have demonstrated that mechanical unloading may result in severe bone loss, as observed in astronauts undergoing long-term space flight or in patients subjected to long-duration immobility or bedrest $(1,2)$. Decreased bone formation caused by abnormal osteoblast function is considered to be one of the primary causes of bone loss (3). Studies have demonstrated that real or simulated microgravity may affect the processes of osteoblast proliferation, differentiation and mineralization (4-6). Genomic analysis revealed that the expression of hundreds of genes in osteoblasts is altered upon exposure to microgravity $(7,8)$. Efforts have been made to identify and characterize the key regulators or genes that control alterations in osteoblasts. However, existing studies have not completely clarified the reason for the delay in osteoblast development induced by microgravity; it is therefore necessary to further examine more regulatory mechanisms, in order to obtain a deep insight into this area and to develop effective preventative measures.

Previously, high throughput genomic analysis and transcriptome sequencing demonstrated that much of the genome is transcribed to noncoding RNAs (ncRNAs) (9). Increasing numbers of ncRNAs, which were previously considered to be transcriptional 'noise', have been observed to be important regulators of gene expression in development, physiology and disease $(10,11)$. A subset of these ncRNAs whose strand length exceeds 200 nucleotides are termed long noncoding RNAs (lncRNAs) (12). Studies have demonstrated that lncRNAs are involved in a range of important cellular processes, including chromatin modification, RNA processing and gene transcription (11). ncRNAs are able to regulate gene expression in close genomic proximity (cis-acting) or target distant transcriptional activators or inhibitors (trans-acting) via diverse mechanisms (13). Such regulatory effects have been additionally observed in the osteogenic differentiation of mesenchymal stem cells (MSCs). IncRNA-maternally expressed 3, which is located near the bone morphogenetic protein 4 (BMP4) gene locus, is able to dissociate the repressor transcription factor SOX-2 from the BMP4 promoter and thereby activate the transcription of BMP4, resulting in the osteogenic differentiation of bone marrow MSCs (14). These previous studies suggested 
that IncRNAs serve an important role upstream of gene expression during normal osteoblast development. However, there are no reports of IncRNA expression patterns in the microgravity-induced inhibition of osteogenic development, and whether mechanical signal-mediated osteoblast differentiation relies on the modulation of IncRNA expression remains unclear.

The present study screened for differentially-expressed lncRNAs and mRNAs in the MC3T3-E1 pre-osteoblast cells, prior to and following exposure to simulated microgravity, using microarray profiling. A series of bioinformatic analyses, including Gene Ontology (GO) analysis, pathway analysis, genomic context analysis and co-expression analysis, were used to predict potential functional lncRNAs. The results of the present study suggested that lncRNAs may serve important roles during osteoblast differentiation under simulated microgravity conditions.

\section{Materials and methods}

Cell culture and osteogenic differentiation. Mouse MC3T3-E1 pre-osteoblast cells were obtained from the Chinese Academy of Sciences Cell Bank (Shanghai, China) and cultured in Dulbecco's modified Eagle's medium(DMEM) containing 10\% fetal bovine serum (FBS) (both from Hyclone; GE Healthcare Life Sciences, Logan, UT, USA), $100 \mathrm{U} / \mathrm{ml}$ penicillin G and $100 \mathrm{mg} / \mathrm{ml}$ streptomycin, at $37^{\circ} \mathrm{C}$ in a humidified atmosphere of $5 \% \mathrm{CO}_{2}$ in air. To induce osteoblast differentiation, the DMEM was supplemented with $10 \%$ FBS, $0.1 \mathrm{mM}$ dexamethasone, $10 \mathrm{mM} \beta$-glycerophosphate, and $50 \mu \mathrm{g} / \mathrm{ml}$ ascorbic acid, and was changed every 2-3 days.

$m R N A$ extraction and reverse transcription-quantitative polymerase chain reaction ( $R T-q P C R$ ) analysis. Total mRNA was extracted using TRIzol reagent (Invitrogen; Thermo Fisher Scientific, Inc., Waltham, MA, USA), according to the manufacturer's instructions. The RNA concentration was quantified using a NanoDrop 1000 Spectrophotometer (Thermo Fisher Scientific, Inc.). RNA quality was tested using an Agilent 2100 bioanalyzer (Agilent Technologies, Inc., Santa Clara, CA, USA) and measured using the RNA integrity number (RIN). RNA samples were submitted for further analysis if the RIN score was $>5.0$.

For RT, first-strand cDNA was synthesized using the PrimeScript ${ }^{\circledR}$ RT reagent kit (cat. no. DRR037; Takara Bio, Inc., Otsu, Japan). The expression levels of target genes were determined quantitatively using a CFX96 qPCR system (Bio-Rad Laboratories, Inc., Hercules, CA, USA) using SYBR ${ }^{\circledR}$ Premix Ex Taq ${ }^{\mathrm{TM}}$ II (cat. no. DRR820A; Takara Bio, Inc.), according to the manufacturer's instructions. The primer pairs were as follows: Runt-related transcription factor 2 (Runx2; GenBank accession no. NM_053470) forward, 5'-CCATAA CGGTCTTCACAAATCC-3' and reverse, 5'-GCGGGACAC CTACTCTCATACT-3'; transcription factor Sp7 (Osx; accession no. NM_001037632) forward, 5'-CAGTAATCTTCGTGC CAGACC-3' and reverse, 5'-CTTCTTTGTGCCTCCTTT TCC-3'; polypyrimidine tract-binding protein 2 (Ptbp2; accession no. NM_001310711) forward, 5'-AAAGTCGCTCTG AGTTGTTAT-3' and reverse, 5'-GCGAAGAGTTTGTCCTC AACC-3'; transportin-1 (Tnpo1; accession no.NM_001048267) forward, 5'-AATTCGCGGTGACTCAGTCTGG-3' and reverse, 5'-TCCATCTTGGTTTGCGAGGC-3'; exostosin-1 (Ext1; accession no. NM_010162) forward, 5'-GAAGAGCAC AGTGGTCGGAA-3' and reverse, 5'-CTCGATGGCCGCTAG AATGT-3'; NONMMUT044983 (NONCODE gene ID NONMMUG027774.1) forward, 5'-CGGCAGGCCTAGTCT TGTAT-3' and reverse, 5'-ACAGCAGAGAGAGCCAAG GA-3'; NONMMUT023474 (gene ID NONMMUG014520.1) forward, 5'-TCTCGAACCCTAGGAGAGCA-3' and reverse, 5'-GGGACAAGGTAAATGGCTCA-3'; NONMMUT018832 (gene ID NONMMUG011720.1) forward, 5'-AACATCTGA GGCTTGGCACT-3' and reverse, 5'-TCATGGTACTGGCAT CTCCA-3'; and GAPDH (accession no. NM_008084) forward, 5'-CAGTGCCAGCCTCGTCTCAT-3' and reverse, 5'-AGG GGCCATCCACAGTCTTC-3'. GAPDH was used as an internal control. The qPCR thermal cycling was performed as follows: Initial incubation for $15 \mathrm{sec}$ at $95^{\circ} \mathrm{C}$, followed by denaturing for 40 cycles at $95^{\circ} \mathrm{C}$ for $5 \mathrm{sec}$ and annealing for $31 \mathrm{sec}$ at $60^{\circ} \mathrm{C}$. The relative expression of target genes was calculated using the $2^{-\Delta \Delta \mathrm{Cq}}$ method (15).

Alkaline phosphatase (ALP) activity assay. Cells were seeded at $1 \times 10^{6}$ cells/well in 6-well plates (Corning Inc., Corning, NY, USA) and cultured for $24 \mathrm{~h}$. When ALP activity was determined, confluent cell layers were washed with PBS, lysed with $0.1 \mathrm{~mol} / 1 \mathrm{M}$-PER mammalian protein extraction reagent (Pierce; Thermo Fisher Scientific, Inc.) for 15-30 min, and centrifuged at $12,000 \mathrm{x} \mathrm{g}$ for $15 \mathrm{~min}$ at room temperature, according to the manufacturer's instructions. The supernatants were collected for determining ALP activity using an ALP assay kit (Nanjing Jiancheng Bioengineering Institute, Nanjing, China). Protein concentrations were measured using a Bicinchoninic Acid Protein Assay kit (Pierce; Thermo Fisher Scientific, Inc.). ALP activity (IU/l) was defined as the release of $1 \mathrm{nmol} p$-nitrophenol $/ \mathrm{min} / \mu \mathrm{g}$ total cellular protein.

Simulated microgravity exposure. The Rotating Wall Vessel Bioreactor (RWVB) clinostat is an effective, ground-based device which is used to simulate microgravity (7). Microgravity is achieved by keeping cells rotating uniformly around a horizontal axis. Therefore, there is a vector-averaged reduction in the apparent gravity acting on the cells when rotated by $360^{\circ}$. A 2D-RWVB (China Astronaut Research and Training Center, Beijing, China) was used in the present study, as previously described (16). MC3T3-E1 cells were seeded on coverslips and incubated until cell confluence reached $70 \%$. The coverslips were subsequently fixed in the bioreactor and placed $12.5 \mathrm{~mm}$ away from the rotational axis. The bioreactor was completely filled with culture medium. Gentle aspiration was performed to clear away air bubbles in order to avoid shear stress during rotation. The bioreactor was fixed onto the clinostat and rotated around a horizontal axis at $24 \mathrm{rpm}$. The group rotating around a vertical axis was regarded as the control. The entire system was placed in a humidified incubator at $37^{\circ} \mathrm{C}$ under $5 \% \mathrm{CO}_{2}$.

Microarray profiling. An Affymetrix GeneChip ${ }^{\circledR}$ Mouse Transcriptome assay (version 1.0; Affymetrix Inc., Santa Clara, CA, USA) provided global profiling of transcripts in the mouse genome, which were selected from the most authoritative databases, including NONCODE 
(www.noncode.org/index.php) and ENSEMBL (www.ensembl.org/index.html). In the microarray, every transcript was detected with 10 specific probes in order to improve the confidence of the statistical results. Protein-coding and non-coding genes were represented on a separate array to supply coincident hybridization. RNA labeling and array hybridization were performed according to the manufacturer's protocol. The microarray work was performed by Shanghai Ming Information Technology Co., Ltd. (Shanghai, China).

GO and pathway analysis. GO analysis was applied to analyze the primary function of the differentially-expressed genes according to the principles of Gene Ontology, which is able to organize genes into hierarchical categories and reveal the gene regulatory network on the basis of biological process and molecular function (17). Pathway analysis was additionally performed to examine the significant pathways of the differentially-expressed genes according to the Kyoto Encyclopedia of Genes and Genomes, Biocarta and Reatome databases (18). An online Bioinformatics enrichment tool (DAVID; david.ncifcrf.gov) was used to perform the GO and pathway analysis in the present study (19). Biological processes of GO terms were illustrated in the GO analysis. The P-value indicated the significance of GO term and pathway term enrichment in the differentially-expressed mRNA list $(\mathrm{P}<0.05$ was considered to be statistically significant).

Construction of the IncRNA-mRNA co-expression network. A co-expression regulatory network is an undirected graph, where each node corresponds to coding or ncRNAs, and a pair of nodes is connected by an edge if there is a significant co-expression association between them (20). IncRNA-mRNA networks were built to identify the interactions between the differentially-expressed genes and lncRNAs, according to the normalized signal intensity of specific expression in the gene and IncRNA. For IncRNA-gene pair, the Pearson correlation was calculated and the significant correlation pairs with which to construct the network were selected (21). In network analysis, degree centrality, which is defined as the number of connections between one node and another, is the simplest and most important measure of the centrality of a gene or lncRNA within a network which determines its relative importance (22).

Statistical analysis. Experiments were repeated a minimum of three times. Data were statistically analyzed using SPSS 19.0 software and expressed as the mean \pm standard deviation. A one-way repeated measures analysis of variance followed by Dunnett's post hoc test was used to compare the time course-dependent variables. Two group comparisons were performed using a two-tailed t-test. $\mathrm{P}<0.05$ was considered to indicate a statistically significant difference.

\section{Results}

Differentially-expressed IncRNAs and mRNAs in MC3T3-E1 cells exposed to simulated microgravity. MC3T3-E1 cells were cultured in an RWVB clinostat and tested following rotation for $72 \mathrm{~h}$. The effect of simulated microgravity on osteoblasts was assessed by observing the gene expression of Runx2 and Osx, in addition to the activity of ALP, which were considered to be important transcription factors for osteoblast differentiation. The data demonstrated that the gene expression of Runx 2 and Osx and the protein activity of ALP were significantly decreased, of which, Osx and ALP reached lowest levels at $48 \mathrm{~h}$ and Runx 2 decreased slightly further at $72 \mathrm{~h}$ (Fig. 1A). The results suggested that osteo-differentiation process of MC3T3-E1 cells was inhibited by simulated microgravity.

In order to detect the differentially-expressed lncRNAs and mRNAs in MC3T3-E1 when exposed to simulated microgravity, a transcriptome assay was performed to compare expression profiles between the normal gravity and simulated microgravity groups. The expression levels of 1,481 ncRNAs were observed to be significantly altered, which is presented in the hierarchical clustering analysis heat map (Fig. 1B). Among them, 857 lncRNAs whose length exceeded 200 nucleotides, including 168 upregulated and 689 downregulated, were screened out and are exhibited on a scatter plot (Fig. 1C). In addition, 2,264 mRNAs were demonstrated to be differentially-expressed, including 459 upregulated mRNAs and 1,805 downregulated mRNAs, presented on a clustering heat map (Fig. 1D).

Construction of the co-expression network between differentially-expressed lncRNAs and mRNAs. Locational distributions of the aberrantly-expressed lncRNAs and mRNAs were analyzed synchronously. Significantly altered lncRNAs and mRNAs were spread across the chromosomes, with the largest amounts on chromosomes 1,2 and 11. A relatively consistent variation pattern was observed between aberrant lncRNA and mRNA species, except for on chromosome 7 (Fig. 2A). In order to elucidate the detailed association, a large and complex lncRNA-mRNA co-expression network was generated according to the normalized signal intensity of differentially-expressed lncRNAs and mRNAs. This co-expression network consisted of 916 nodes and 4,813 connections between 354 lncRNAs and 562 mRNAs. Within this network, there were 3,798 pairs presenting as positive regulatory associations and 1,015 pairs as negative associations. Subsequently, mRNAs in the network were submitted for GO enrichment analysis. The results demonstrated that the aberrant mRNAs were frequently enriched in such biological process as phosphorylation, response to DNA damage, the cell cycle, regulation of gene expression and cell proliferation (Fig. 2B), and were involved in the cell signaling pathways of the cell cycle, focal adhesion, cancer and viral infection (Fig. 2C).

Genomic context analysis of the aberrantly-expressed IncRNAs. In order to further elicit information from the co-expression network and examine the possible functional lncRNAs, the nearby mRNAs located within $100 \mathrm{~kb}$ up or downstream of aberrantly-expressed lncRNAs were identified and matched with the aberrantly-expressed mRNAs in the microarray data. Among them, 132 differentially-expressed lncRNAs and nearby mRNAs pairs were identified (data not shown). GO analysis indicated that the nearby genes were involved in the significant biological processes, including the cell cycle, nervous system development, response to growth 

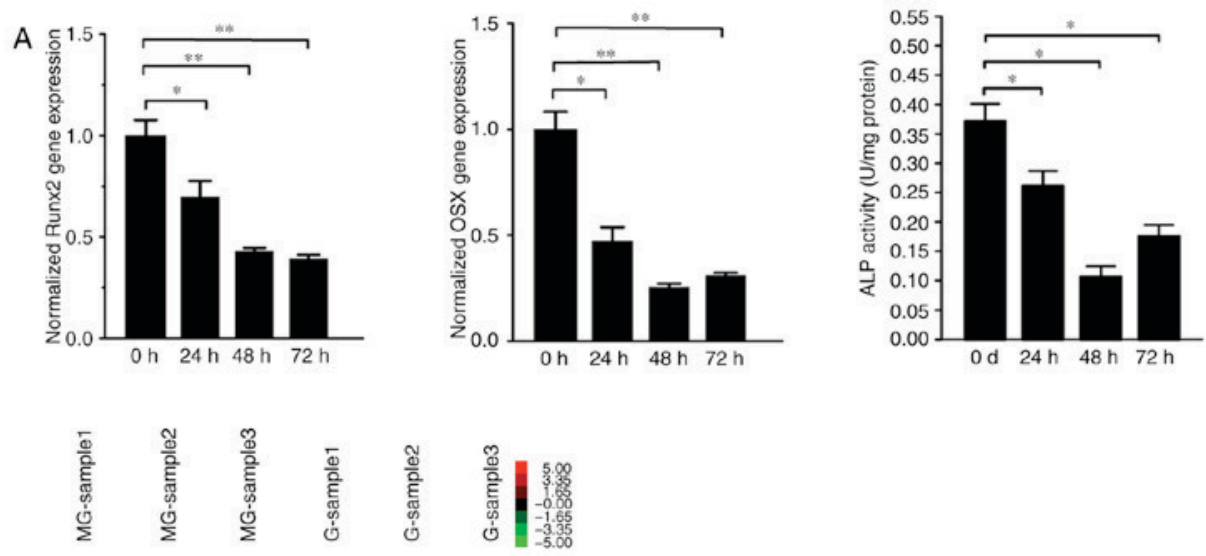

B
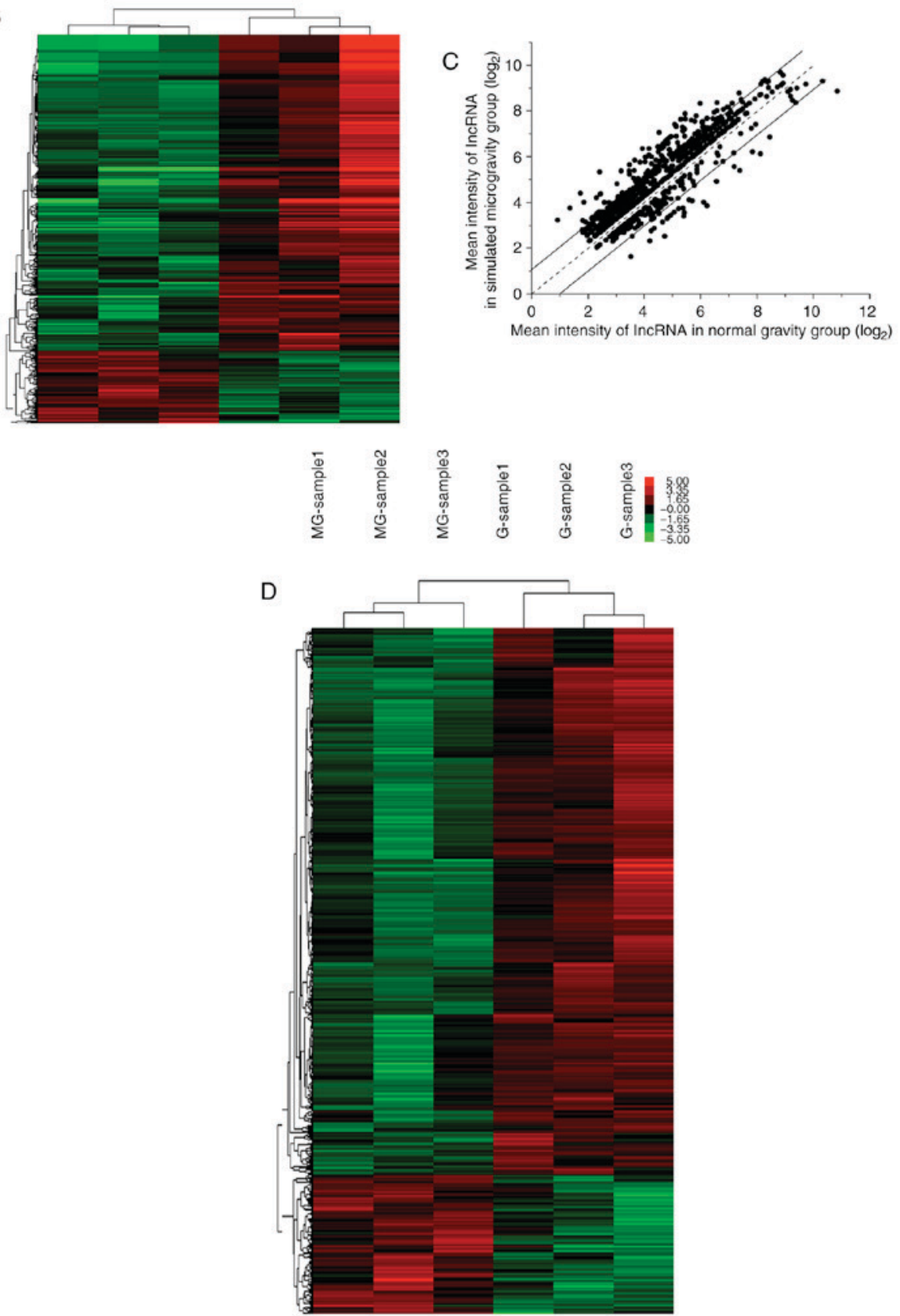

Figure 1. Expression profile of lncRNAs and mRNAs in MC3T3-E1 cells exposed to simulated microgravity. (A) Osteogenic differentiation was assessed by reverse transcription-quantitative polymerase chain reaction analysis of osteoblast marker genes and activity analysis of ALP. $n=3$. ${ }^{*} \mathrm{P}<0.05,{ }^{* * *} \mathrm{P}<0.01$. (B) Expression profiles of ncRNAs in MC3T3-E1 cells when exposed to simulated microgravity. Green represents downregulated ncRNAs, and red represents upregulated ncRNAs. $n=3$. (C) The deregulated lncRNAs with a length exceeding 200 nucleotides were screened out and exhibited on a scatter plot. (D) Expression profile of mRNAs in MC3T3-E1 cells when exposed to simulated microgravity. $n=3$. IncRNA, long noncoding RNA; ALP, alkaline phosphatase; Runx2, runt-related transcription factor 2; OSX, transcription factor Sp7. 

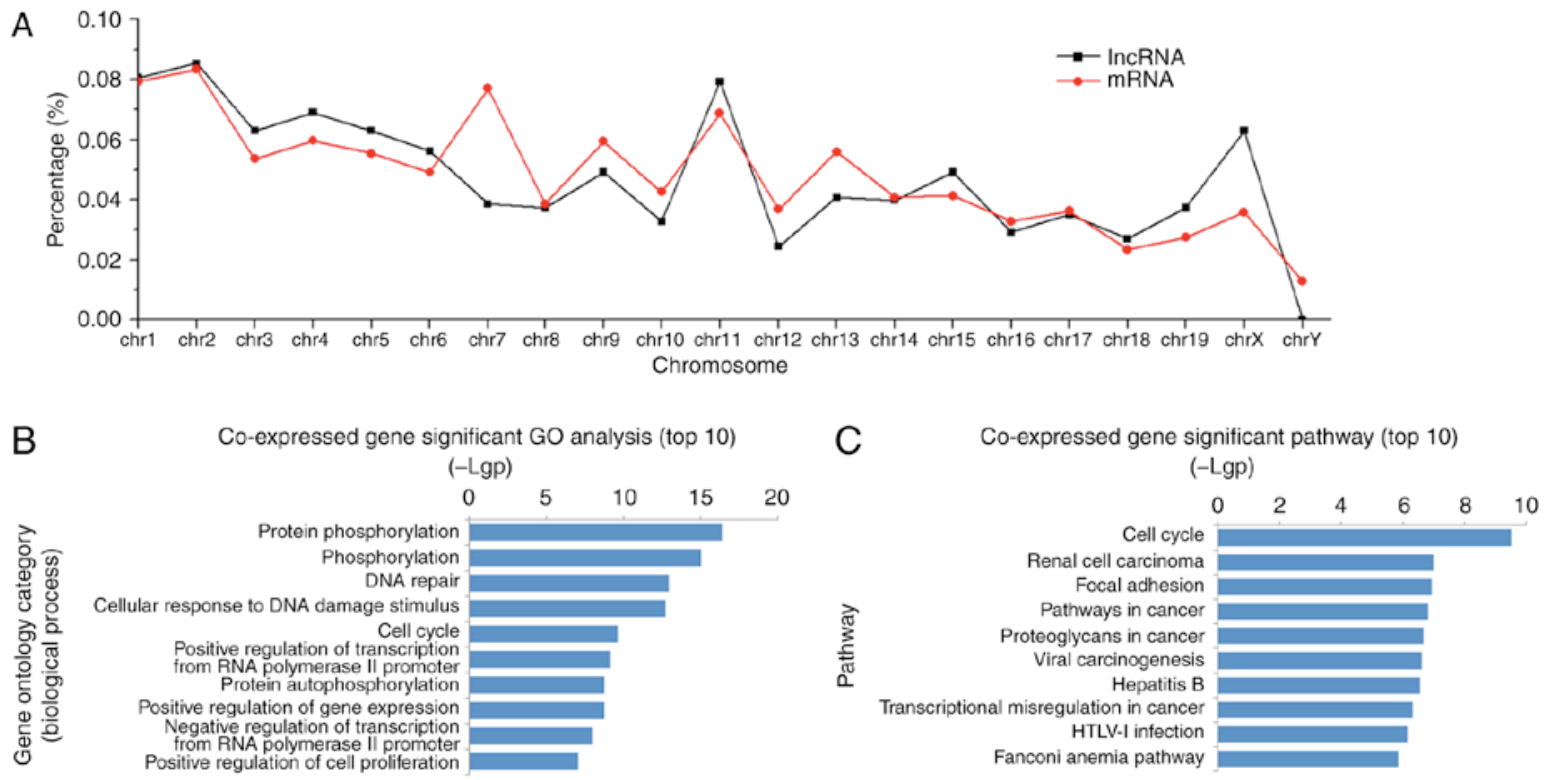

Figure 2. Chromosome location, GO and pathway analyses of deregulated lncRNAs and mRNAs. (A) Location distributions of deregulated lncRNAs and mRNAs on chromosomes. The co-expressed genes of deregulated lncRNAs were submitted for GO and pathway enrichment analyses. (B) The top 10 enriched GO terms (biological process) and (C) signal pathways are presented in bar diagrams. The value of $-\log _{10}$ (P-value) was calculated to reflect the significance of enrichment. GO, Gene Ontology; lncRNA, long noncoding RNA.

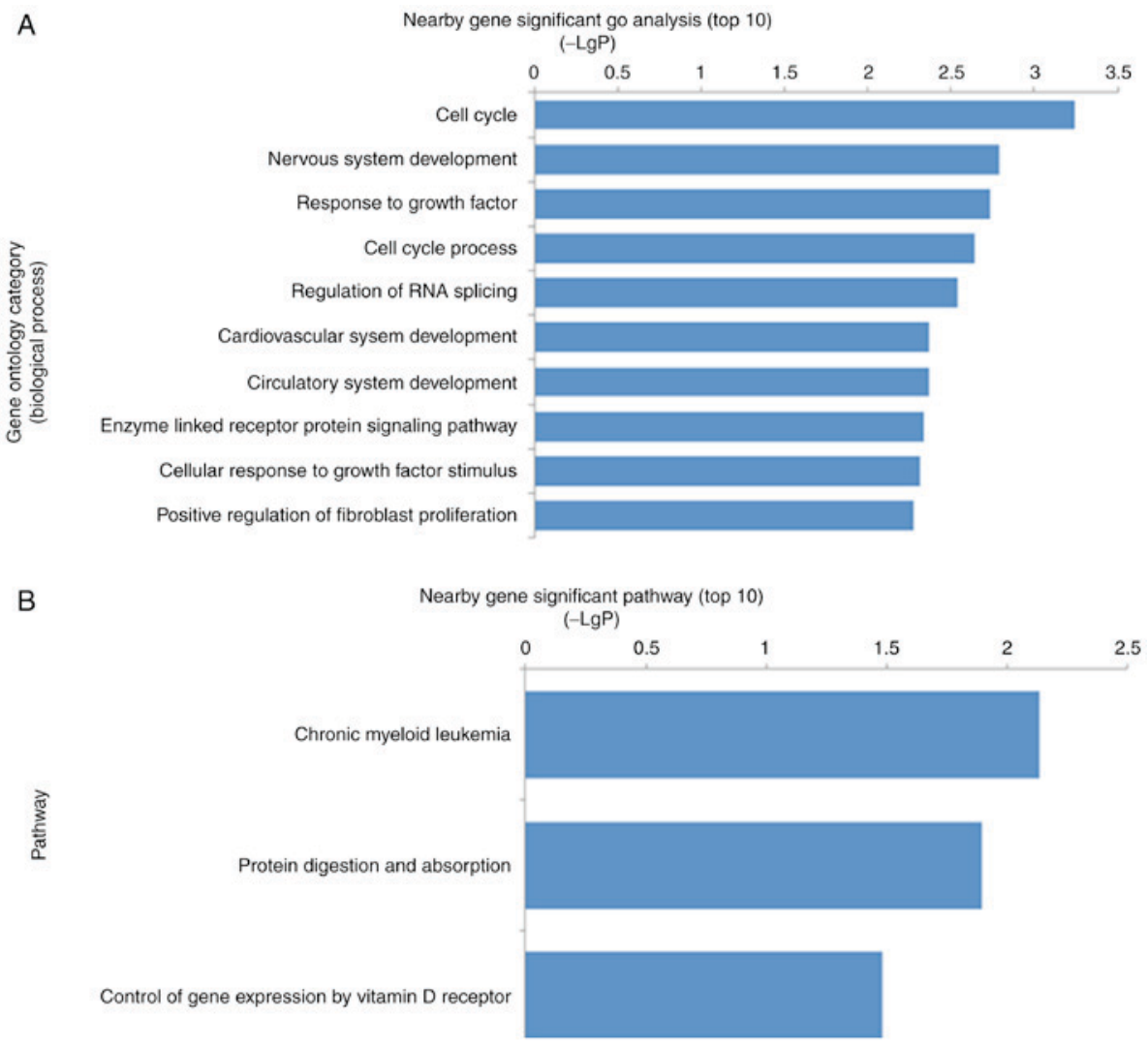

Figure 3. GO and pathway analyses of genes located near the deregulated long noncoding RNAs. (A) The top 10 enriched GO terms (biological process). (B) The top 10 enriched signal pathways. GO, Gene Ontology.

factors, regulation of RNA splicing, circulatory system development, enzyme-linked receptor protein signaling pathway and positive regulation of fibroblast proliferation (Fig. 3A). Pathway analysis demonstrated that the most significant pathways were chronic myeloid leukemia, protein digestion and absorption, and control of gene expression by vitamin D receptor (Fig. 3B). A literature review further demonstrated that $\geq 15$ mRNAs included in 17 lncRNA-mRNA pairs have been reported to be involved in the regulation of osteoblast proliferation, differentiation and mineralization (Table I). 


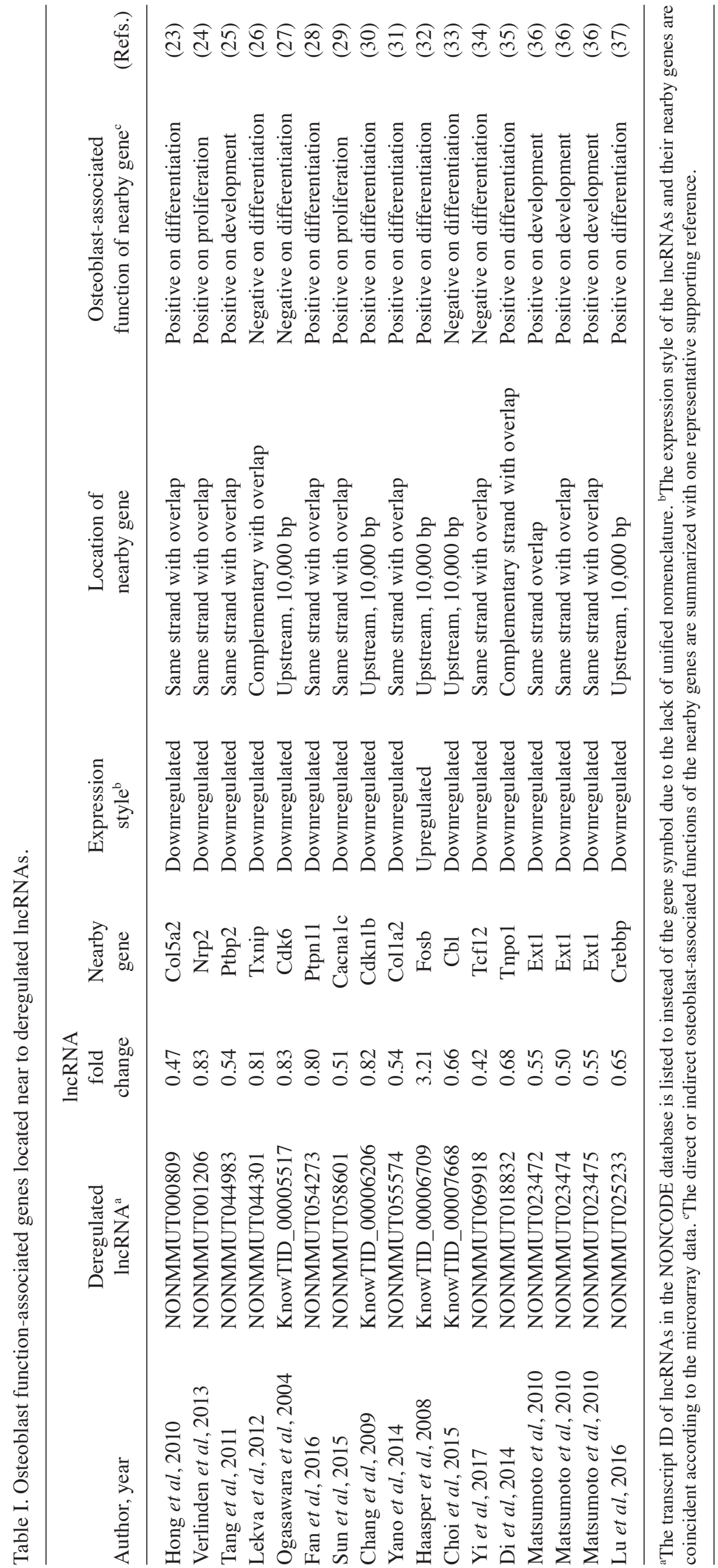


A

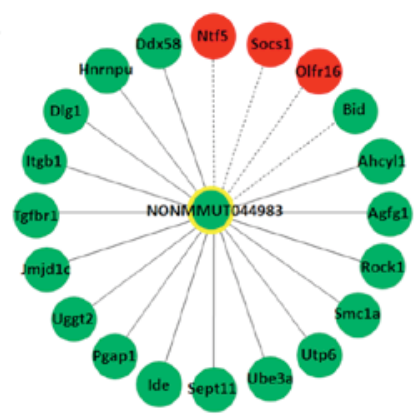

B

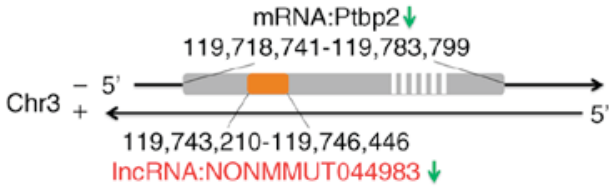

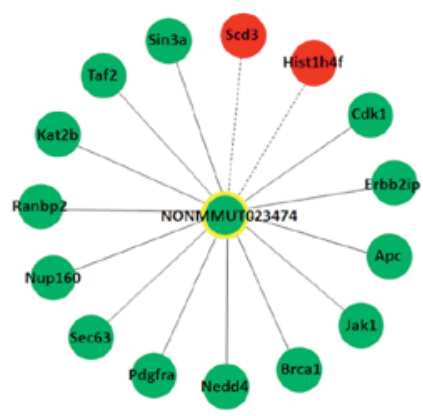

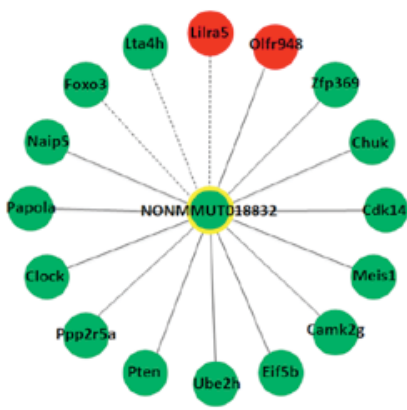

mRNA:Tnpo1 $\downarrow$

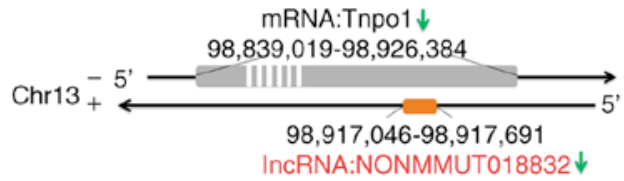

mRNA:Ext1 $\downarrow$
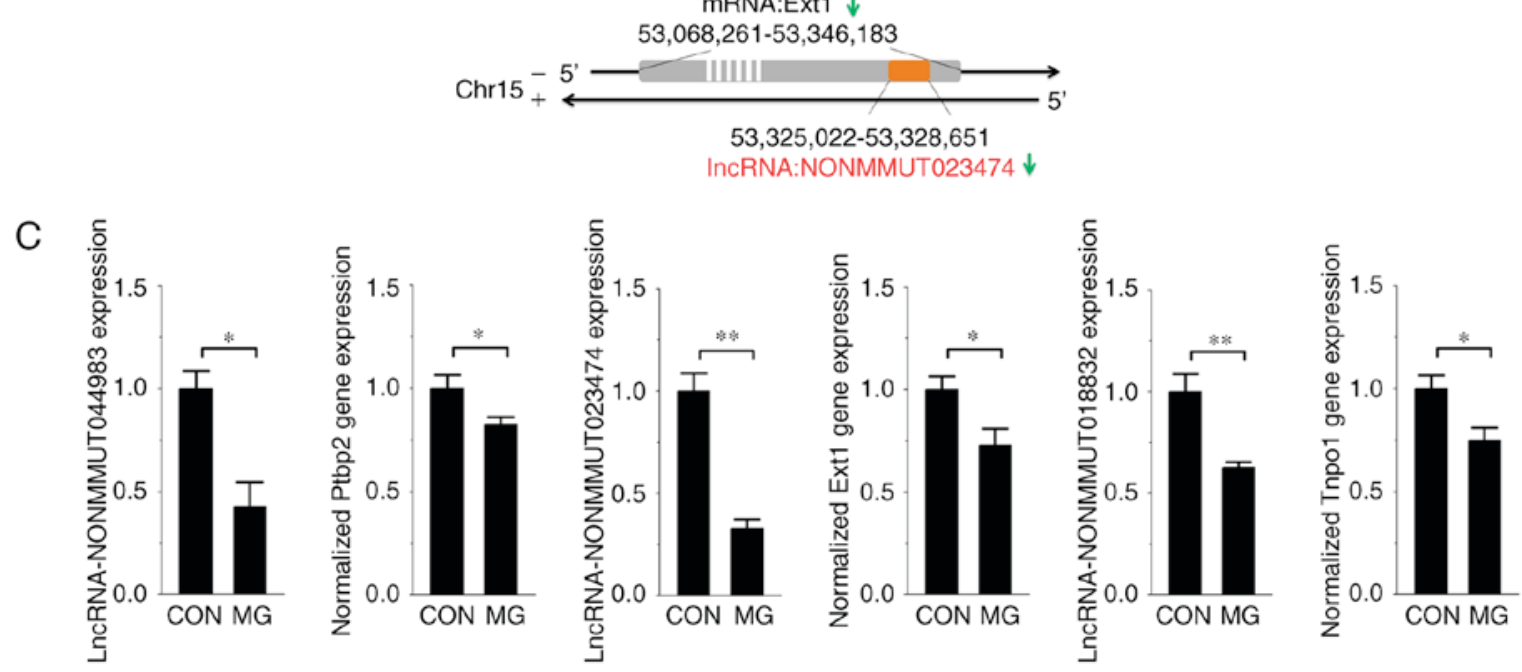

Figure 4. Association between target lncRNAs and co-expressed or nearby genes. (A) The co-expression network was rebuilt between the target lncRNAs and their co-expressed deregulated mRNAs. Red nodes represent upregulated genes and green nodes represent downregulated genes. A solid line between two nodes represents a positive association while a dotted line represents a negative association. (B) The positional association on the chromosome of the target lncRNAs and their adjacent protein-coding genes. (C) Reverse transcription-quantitative polymerase chain reaction analysis of lncRNAs and their nearby genes in MC3T3-E1 cells exposed to simulated microgravity for $48 \mathrm{~h} . \mathrm{n}=3$. ${ }^{*} \mathrm{P}<0.05,{ }^{* *} \mathrm{P}<0.01$. lncRNA, long noncoding RNA; Ptbp2, polypyrimidine tract-binding protein 2; Ext1, exostosin-1; Tnpo1, transportin-1; CON, control; MG, microgravity.

Bioinformatics screening of potential functional lncRNAs. In the co-expression network, degree centrality was considered to be the simplest and most important measure by which to estimate the core regulatory factors. In order to obtain more accurate candidate IncRNAs, the degree centrality of the 17 lncRNAs whose nearby genes had been reported to be associated with osteoblast function was calculated. The IncRNAs NONMMUT044983, NONMMUT023474 and NONMMUT018832 were screened out via the degree centrality analysis (degree $>15$ ). Their associations with deregulated mRNAs were obtained from the overall co-expression network and rebuilt (Fig. 4A). The locations of the lncRNAs compared with mRNAs on the chromosome are exhibited in Fig. 4B. In addition, RT-qPCR analysis was performed to determine the expression of the three lncRNAs and their nearby genes in MC3T3-E1 cells exposed to the RWVB clinostat for $48 \mathrm{~h}$. The results of the present study demonstrated that the expression of each lncRNA and mRNA was significantly decreased under simulated microgravity (Fig. 4C), consistent with the microarray data.

\section{Discussion}

Osteoblasts, the main functional cells of bone formation, have been reported to be associated with the bone loss induced by microgravity $(38,39)$. A number of important regulators or genes have been studied to examine how microgravity may affect osteoblast function. In the present study, global genome analysis was performed and it was observed that $857 \mathrm{lncRNAs}$ and 2,264 mRNAs were significantly altered in MC3T3-E1 cells exposed to simulated microgravity. Bioinformatic analyses were performed to identify potential functional lncRNAs. To the best of our knowledge, the present study is the first to suggest that lncRNAs may serve important roles in osteoblast differentiation under simulated microgravity conditions and provide a novel perspective on the effects of microgravity on osteoblast function.

Previous studies have revealed that a number of lncRNAs are not transcriptional noise and serve important regulatory roles $(11,13)$. However, unlike the relatively definite regulatory mechanisms of miRNAs, lncRNA function may not 
be accurately predicted from the sequence or structure. A previous study demonstrated that the potential function of lncRNA may be inferred from their co-expressed genes (40). In the present study, a relatively consistent variation pattern, except for on chromosome 7 , was observed between the differentially-expressed lncRNAs and mRNAs, suggesting that they were more co-expressed or inversely-expressed than may be expected by chance. The co-expression regulatory network was built according to the normalized signal intensity of the differentially-expressed lncRNAs and mRNAs. The co-expressed IncRNAs/genes in the network were hypothesized to be controlled by the same transcriptional regulatory pathway, to be functionally similar, or to be members of the same pathway or protein complex (41). Therefore, the functions of the deregulated lncRNAs may cover the biological process of the cell cycle, regulation of gene expression or cell proliferation, which were inferred from functional enrichment analysis of their co-expressed genes.

Apart from expression-based approaches, the genomic context of lncRNAs is frequently studied in order to elucidate their functions. An important localized regulatory mechanism through which lncRNAs may affect the expression of nearby protein-coding genes has been confirmed $(42,43)$. For example, lncRNA-Evf2, generated from the homeobox protein Dlx-5/6 ultra conserved region, is able to act concurrently with the transcription factor Dlx 2 to increase the transcriptional activity of the Dlx-5/6 enhancer in a target- and homeodomain-specific manner (43). Studying the nearby coding mRNAs may enhance the understanding of the function and potential regulatory mechanisms of lncRNAs. In the present study, 132 differentially-expressed lncRNAs and their nearby coding mRNA pairs were identified. The 132 differentially-expressed mRNAs were involved in the significant biological processes of the cell cycle, nervous system development and response to growth factors, or in the signaling pathways of chronic myeloid leukemia, protein digestion and absorption, and control of gene expression by vitamin $\mathrm{D}$ receptor. In particular, the vitamin D receptor pathway is associated with osteogenic development (44). Certain deregulated lncRNAs were more likely to affect osteoblast function by acting on their nearby osteoblast-associated mRNAs. Notably, 15 of the nearby mRNAs have been previously reported to be involved in osteoblast development. The corresponding 17 lncRNAs are therefore important candidates for examining the function of lncRNAs in osteoblast development.

To identify the most likely functional lncRNAs, degree centrality within the co-expression network was considered. Larger degree centrality values indicate an increased possibility of a regulatory role. Therefore, the IncRNAs NONMMUT044983, NONMMUT018832 and NONMMUT023474 were screened out via degree centrality at the forefront. According to the degree of functional association and similarity between co-expression genes, it may be possible to infer the potential function of these lncRNAs from their co-expressed genes, although GO analysis was not feasible due to the insufficient sample size. Alternative analyses of the functions of the three IncRNAs focused on the nearby genes. Their nearby coding mRNAs exhibited an association with osteoblast development, according to previous literature. Ptbp2 regulates the mutually exclusive exons $8 \mathrm{a}$ and 8 in the
CaV1.2 calcium channel transcript (25), and the latter serves fundamental roles in cellular responses to external stimuli, including mechanical forces and hormonal signals, in osteoblastic lineage bone cells $(45,46)$. Tnpol is able to facilitate the nuclear translocation of oxytocin receptors and result in osteoblast maturation. The knockdown of Tnpol may abrogate the oxytocin-induced expression of osteoblast differentiation genes Osx, cyclic AMP-dependent transcription factor Atf4 and osteocalcin (35). In addition, it has been reported that conditional ablation of Ext1 may lead to dysregulation of BMP signaling and severe skeletal defects (36). Microarray and RT-qPCR analyses suggested that the expression of the three lncRNAs and their nearby genes were significantly decreased under simulated microgravity. Therefore, it was hypothesized that downregulation of these IncRNAs may abrogate the expression of their nearby osteoblast-associated genes and result in inhibited osteogenesis under simulated microgravity. Future studies are required to investigate the potential regulatory mechanisms.

In conclusion, 857 differentially-expressed lncRNAs were identified in the present study when the osteo-differentiating MC3T3-E1 cells were exposed to simulated microgravity. A number of potential functional lncRNAs, and the possible regulatory mechanisms through which lncRNAs may control osteoblast differentiation in a microgravity environment were predicted using bioinformatics analysis. Although further experiment studies are required to test this hypothesis, the results of the present study demonstrated that lncRNAs are novel and potent candidates for studies into the effects and mechanism of microgravity in osteoblast function.

\section{Acknowledgements}

The present study was supported by grants from the National Natural Science Foundation of China (grant nos. 31570939, 81701856, 81471815 and 31170889).

\section{References}

1. Bloomfield SA: Disuse osteopenia. Curr Osteoporos Rep 8: 91-97, 2010

2. Ohshima H: Secondary osteoporosis UPDATE. Bone loss due to bed rest and human space flight study. Clin Calcium 20: 709-716, 2010 (In Japanese).

3. Morey ER and Baylink DJ: Inhibition of bone formation during space flight. Science 201: 1138-1141, 1978.

4. Zayzafoon M, Gathings WE and McDonald JM: Modeled microgravity inhibits osteogenic differentiation of human mesenchymal stem cells and increases adipogenesis. Endocrinology 145: 2421-2432, 2004.

5. Rodionova NV: The dynamics of proliferation and differentiation of osteogenic cells under supportive unloading. Tsitol Genet 45: 22-27, 2011

6. Hu LF, Li JB, Qian AR, Wang F and Shang P: Mineralization initiation of MC3T3-E1 preosteoblast is suppressed under simulated microgravity condition. Cell Biol Int 39: 364-372, 2015.

7. Capulli M, Rufo A, Teti A and Rucci N: Global transcriptome analysis in mouse calvarial osteoblasts highlights sets of genes regulated by modeled microgravity and identifies a "mechanoresponsive osteoblast gene signature'. J Cell Biochem 107: 240-252, 2009

8. Patel MJ, Liu W, Sykes MC, Ward NE, Risin SA, Risin D and Jo H: Identification of mechanosensitive genes in osteoblasts by comparative microarray studies using the rotating wall vessel and the random positioning machine. J Cell Biochem 101: $587-599,2007$. 
9. Claverie JM: Fewer genes, more noncoding RNA. Science 309: 1529-1530, 2005.

10. Kapranov P, Cheng J, Dike S, Nix DA, Duttagupta R, Willingham AT, Stadler PF, Hertel J, Hackermüller J, Hofacker IL, et al: RNA maps reveal new RNA classes and a possible function for pervasive transcription. Science 316: 1484-1488, 2007.

11. Mercer TR, Dinger ME and Mattick JS: Long noncoding RNAs: Insights into functions. Nat Rev Genet 10: 155-159, 2009.

12. Brosnan CA and Voinnet O: The long and the short of noncoding RNAs. Curr Opin Cell Biol 21: 416-425, 2009.

13. Ponting CP, Oliver PL and Reik W: Evolution and functions of long noncoding RNAs. Cell 136: 629-641, 2009.

14. Zhuang W, Ge X, Yang S, Huang M, Zhuang W, Chen P, Zhang X, Fu J, Qu J and Li B: Upregulation of lncRNA MEG3 promotes osteogenic differentiation of mesenchymal stem cells from multiple myeloma patients by targeting BMP4 transcription. Stem Cells 33: 1985-1997, 2015.

15. Livak KJ and Schmittgen TD: Analysis of relative gene expression data using real-time quantitative PCR and the 2(-Delta Delta C(T)) method. Methods 25: 402-408, 2001.

16. Hu Z, Wang Y, Sun Z, Wang H, Zhou H, Zhang L, Zhang S and Cao X: miRNA-132-3p inhibits osteoblast differentiation by targeting Ep300 in simulated microgravity. Sci Rep 5: 18655, 2015.

17. Ashburner M, Ball CA, Blake JA, Botstein D, Butler $\mathrm{H}$, Cherry JM, Davis AP, Dolinski K, Dwight SS, Eppig JT, et al: Gene ontology: Tool for the unification of biology. The gene ontology consortium. Nat Genet 25: 25-29, 2000

18. Draghici S, Khatri P, Tarca AL, Amin K, Done A, Voichita C, Georgescu C and Romero R: A systems biology approach for pathway level analysis. Genome Res 17: 1537-1545, 2007.

19. Huang da W, Sherman BT and Lempicki RA: Systematic and integrative analysis of large gene lists using DAVID bioinformatics resources. Nat Protoc 4: 44-57, 2009.

20. Stuart JM, Segal E, Koller D and Kim SK: A gene-coexpression network for global discovery of conserved genetic modules Science 302: 249-255, 2003.

21. Prieto C, Risueño A, Fontanillo $C$ and De las Rivas J: Human gene coexpression landscape: Confident network derived from tissue transcriptomic profiles. PLoS One 3: e3911, 2008.

22. Barabási AL and Oltvai ZN: Network biology: Understanding the cell's functional organization. Nat Rev Genet 5: 101-113, 2004

23. Hong D, Chen HX, Yu HQ, Liang Y, Wang C, Lian QQ, Deng HT and Ge RS: Morphological and proteomic analysis of early stage of osteoblast differentiation in osteoblastic progenitor cells. Exp Cell Res 316: 2291-2300, 2010

24. Verlinden L, Kriebitzsch C, Beullens I, Tan BK, Carmeliet G and Verstuyf A: Nrp2 deficiency leads to trabecular bone loss and is accompanied by enhanced osteoclast and reduced osteoblast numbers. Bone 55: 465-475, 2013.

25. Tang ZZ, Sharma S, Zheng S, Chawla G, Nikolic J and Black DL: Regulation of the mutually exclusive exons $8 \mathrm{a}$ and 8 in the CaV1.2 calcium channel transcript by polypyrimidine tract-binding protein. J Biol Chem 286: 10007-10016, 2011.

26. Lekva T, Ueland T, Bøyum H, Evang JA, Godang K and Bollerslev J: TXNIP is highly regulated in bone biopsies from patients with endogenous Cushing's syndrome and related to bone turnover. Eur J Endocrinol 166: 1039-1048, 2012.

27. Ogasawara T, Kawaguchi H, Jinno S, Hoshi K, Itaka K, Takato T, Nakamura K and Okayama H: Bone morphogenetic protein 2 -induced osteoblast differentiation requires Smad-mediated down-regulation of Cdk6. Mol Cell Biol 24: 6560-6568, 2004.

28. Fan D, Liu S, Jiang S, Li Z, Mo X, Ruan H, Zou GM and Fan C: The use of SHP-2 gene transduced bone marrow mesenchymal stem cells to promote osteogenic differentiation and bone defect repair in rat. J Biomed Mater Res A 104: 1871-1881, 2016.

29. Sun Z, Cao X, Hu Z, Zhang L, Wang H, Zhou H, Li D, Zhang S and Xie M: miR-103 inhibits osteoblast proliferation mainly through suppressing Cav1.2 expression in simulated microgravity. Bone 76: 121-128, 2015
30. Chang SF, Chang TK, Peng HH, Yeh YT, Lee DY, Yeh CR, Zhou J, Cheng CK, Chang CA and Chiu JJ: BMP-4 induction of arrest and differentiation of osteoblast-like cells via p21 CIP1 and p27 KIP1 regulation. Mol Endocrinol 23: 1827-1838, 2009.

31. Yano H, Hamanaka R, Nakamura-Ota M, Adachi S, Zhang JJ, Matsuo $\mathrm{N}$ and Yoshioka H: Sp7/Osterix induces the mouse pro- $\alpha 2$ (I) collagen gene (Colla2) expression via the proximal promoter in osteoblastic cells. Biochem Biophys Res Commun 452: 531-536, 2014

32. Haasper C, Jagodzinski M, Drescher M, Meller R, Wehmeier M, Krettek C and Hesse E: Cyclic strain induces FosB and initiates osteogenic differentiation of mesenchymal cells. Exp Toxicol Pathol 59: 355-363, 2008

33. Choi YH, Han Y, Lee SH, Jin YH, Bahn M, Hur KC, Yeo CY and Lee KY: Cbl-b and c-Cbl negatively regulate osteoblast differentiation by enhancing ubiquitination and degradation of Osterix. Bone 75: 201-209, 2015.

34. Yi S, Yu M, Yang S, Miron RJ and Zhang Y: Tcf12, A member of basic helix-loop-helix transcription factors, mediates bone marrow mesenchymal stem cell osteogenic differentiation in vitro and in vivo. Stem Cells 35: 386-397, 2017.

35. Di Benedetto A, Sun L, Zambonin CG, Tamma R, Nico B, Calvano CD, Colaianni G, Ji Y, Mori G, Grano M, et al: Osteoblast regulation via ligand-activated nuclear trafficking of the oxytocin receptor. Proc Natl Acad Sci USA 111: 16502-16507, 2014.

36. Matsumoto Y, Matsumoto K, Irie F, Fukushi J, Stallcup WB and Yamaguchi Y: Conditional ablation of the heparan sulfate-synthesizing enzyme Ext1 leads to dysregulation of bone morphogenic protein signaling and severe skeletal defects. J Biol Chem 285: 19227-19234, 2010.

37. Lu J, Qu S, Yao B, Xu Y, Jin Y, Shi K, Shui Y, Pan S, Chen L and Ma C: Osterix acetylation at K307 and K312 enhances its transcriptional activity and is required for osteoblast differentiation. Oncotarget 7: 37471-37486, 2016.

38. Kacena MA, Todd P and Landis WJ: Osteoblasts subjected to spaceflight and simulated space shuttle launch conditions. In Vitro Cell Dev Biol Anim 39: 454-459, 2003.

39. Bucaro MA, Fertala J, Adams CS, Steinbeck M, Ayyaswamy P, Mukundakrishnan K, Shapiro IM and Risbud MV: Bone cell survival in microgravity: Evidence that modeled microgravity increases osteoblast sensitivity to apoptogens. Ann N Y Acad Sci 1027: 64-73, 2004.

40. Necsulea A, Soumillon M, Warnefors M, Liechti A, Daish T, Zeller U, Baker JC, Grützner F and Kaessmann H: The evolution of lncRNA repertoires and expression patterns in tetrapods. Nature 505: 635-640, 2014.

41. Weirauch Matthew T: Gene coexpression networks for the analysis of DNA microarray data. In: Applied Statistics for Network Biology: Methods in Systems Biology. Dehmer M, Emmert-Streib F, Graber A and Salvador A (eds). Wiley-VCH Verlag GmbH \& Co. KGaA, Weinheim, Germany, 2011.

42. Martianov I, Ramadass A, Serra Barros A, Chow N and Akoulitchev A: Repression of the human dihydrofolate reductase gene by a non-coding interfering transcript. Nature 445: 666-670, 2007.

43. Feng J, Bi C, Clark BS, Mady R, Shah P and Kohtz JD: The Evf-2 noncoding RNA is transcribed from the Dlx-5/6 ultraconserved region and functions as a Dlx-2 transcriptional coactivator. Genes Dev 20: 1470-1484, 2006

44. Olivares-Navarrete R, Sutha K, Hyzy SL, Hutton DL, Schwartz Z, McDevitt T and Boyan BD: Osteogenic differentiation of stem cells alters vitamin D receptor expression. Stem Cells Dev 21: 1726-1735, 2012

45. Duncan RL, Akanbi KA and Farach-Carson MC: Calcium signals and calcium channels in osteoblastic cells. Semin Nephrol 18: 178-190, 1998.

46. Iqbal J and Zaidi M: Molecular regulation of mechanotransduction. Biochem Biophys Res Commun 328: 751-755, 2005. 